\title{
Combined reconstructive and deconstructive endovascular approach for bilateral vertebral artery dissection with subarachnoid hemorrhage
}

Jacob Cherian, MD, Thomas P. Madaelil, MD, Frank Tong, MD, Brian M. Howard, MD, C. Michael Cawley, MD, and Jonathan A. Grossberg, MD

Department of Neurosurgery, Emory University Hospital, Atlanta, Georgia

The video highlights a challenging case of bilateral vertebral artery dissection presenting with subarachnoid hemorrhage. The patient was found to have a critical flow-limiting stenosis in his dominant right vertebral artery and a ruptured pseudoaneurysm in his left vertebral artery. A single-stage endovascular treatment with stent reconstruction of the right vertebral artery and coil embolization sacrifice of the left side was performed. The case highlights the rationale for treatment and potential alternative strategies.

The video can be found here: https://youtu.be/e0U_JE2jISw.

KEYWORDS vertebral artery dissection; stenting; sacrifice; video 